\section{Measurement of full-thickness macular hole size using en face optical coherence tomography}

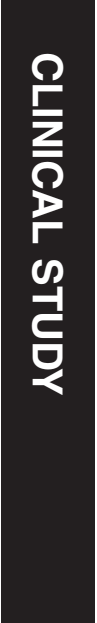

Department of

Ophthalmology, Hôpital

Lariboisière, Université Paris

7- Sorbonne Paris Cité, Paris, France

\section{Correspondence:}

E Philippakis, Department of Ophthalmology, Hôpital Lariboisière, 2 rue Ambroise Paré, Paris Cedex 10 75475, France

Tel: +33149952523;

Fax: +33149956483.

E-mail: elise.philippakis@ aphp.fr

Received: 9 March 2017 Accepted in revised form: 16 October 2017

Published online:

8 December 2017

\author{
Abstract \\ Purpose To assess the feasibility and \\ interest of measuring macular hole (MH) size \\ using en face optical coherence tomography \\ (OCT) compared with manual diameter \\ measurements on B-scans. \\ Methods Among our previously published \\ series of $\mathbf{1 0 0}$ patients operated for primary \\ MH, patients whose images were acquired \\ with Cirrus 5000 HD-OCT (Carl Zeiss \\ Meditec) with a quality signal strength $>5 / 10$ \\ were included. Three segmentations (internal \\ limiting membrane, horizontal, and retinal \\ pigment epithelium (RPE)) were compared \\ for obtaining the most appropriate en face \\ image. MH surface areas were measured \\ using ImageJ software. Mean diameters \\ calculated from surface areas \\ (diameter $=2 \times \sqrt{ }($ surface area $/ \pi))$ were \\ compared with those measured on B-scans. \\ Results Nineteen patients were included \\ with a mean age of $72 \pm 8$ years (56-86) and a \\ female predominance $(3 / 16)$. The mean \\ absolute difference between horizontal and \\ vertical diameters measured on B-scans was \\ of $54 \pm 47 \mu \mathrm{m}(0-180)$ without reaching \\ significance $(P=0.874)$. RPE segmentation \\ provided the best en face image and was \\ feasible without and with adjustment, \\ respectively, in $79 \%$ and $100 \%$ (cases with \\ vitreomacular traction) of cases. No significant \\ difference in mean diameters was observed \\ between those calculated from en face images \\ $(435 \pm 143 \mu \mathrm{m} \mathrm{(195-693))}$ and those measured \\ on horizontal B-scans $(426 \pm 139 \mu \mathrm{m}(214-705)$, \\ $P=0.482$ ). \\ Conclusion Measuring MH size on en face \\ OCT images is feasible, reliable, and \\ eliminates the potential bias related to \\ manual measurements on B-scans. Its \\ integration into OCT devices would offer an \\ automated and easy-to-use option for clinical \\ practice.
}

E Philippakis, M Legrand, M El Sanharawi, A Erginay, A Couturier and R Tadayoni
Eye (2018) 32, 590-596; doi:10.1038/eye.2017.254; published online 8 December 2017

Introduction

Full-thickness macular hole (MH) description and classifications include their size, from Gass classification to the recent classification of the International Vitreomacular Traction Study Group (IVTSG). ${ }^{1,2} \mathrm{MH}$ size influences the therapeutic approach, from enzymatic vitreolysis to surgery, ${ }^{3,4}$ and is also a prognostic factor for both anatomical and functional outcomes. $^{5-7}$ According to the IVTSG, MH size is defined by the aperture size. Then, the size has been reduced to the $\mathrm{MH}$ diameter that is commonly measured manually with a caliper often on the horizontal B-scan of SpectralDomain optical coherence tomography (OCT) passing through the fovea center. ${ }^{2}$ This manual method presents several limitations, from the type of acquisition to the observer's experience. The current challenge is to propose an automated method for $\mathrm{MH}$ size measurement. En face OCT C-scan is a modality that facilitates the transversal assessment of retinal structures such as epiretinal folds or retinal pigment epithelium (RPE) mottling. ${ }^{8-10}$ This visualization technique provides a bird's eye view over the $\mathrm{MH}$, more similar to what is observed on fundus examination. ${ }^{10,11} \mathrm{MH}$ presents as a

hyporeflective cavity surrounded by the highcontrast boundaries of the retina, the RPE and internal limiting membrane (ILM), providing clean-cut boundaries that are easy-to analyze on a computer. Therefore, MHs are well suited for automated segmentation. The aim of this study was to propose a feasible and reliable automated measurement method of $\mathrm{MH}$ size through en face OCT C-scan and to determine whether it was in line with the reference technique. 


\section{Materials and methods}

This retrospective observational case series met the tenets of the Declaration of Helsinki. The ethics committee of the French Society of Ophthalmology approved the conduct of this retrospective review of patient records (IRB 00008855 Société Française d'Ophtalmologie IRB\#1). Patient consent was obtained. The records of a previously published series of 100 consecutive patients operated for primary MH in 2012 in the Department of Ophthalmology of Lariboisière Hospital, Paris, France were retrospectively reviewed. ${ }^{12}$ Patients who had been imaged with the Cirrus 5000 HD-OCT (version 5.0; Carl Zeiss Meditec, Dublin, CA, USA) allowing en face analysis were included. To prevent any bias due to poor quality images, only macular cube images with a signal strength $>5 / 10$ were analyzed. Patients had undergone ophthalmologic examination, including full medical history, best-corrected visual acuity, slit-lamp examination, fundoscopy, and color retinography.

\section{Measurements of MH diameters on OCT B-scans}

Acquired images included a macular cube of $6 \times 6 \mathrm{~mm}$ (128 B-scans $\times 512 \mathrm{~A}$-scans, spacing of $47 \mu \mathrm{m}$ ) and two high-definition five raster lines $(9 \mathrm{~mm}$ width horizontally and vertically, spacing of $75 \mu \mathrm{m})$. In accordance with the reference measurement technique, $\mathrm{MH}$ diameters were manually measured on the horizontal and vertical B-scans passing through the fovea center, at the narrowest area between $\mathrm{MH}$ edges, using a caliper placed on a line manually drawn roughly parallel to the RPE (Figure 1). This measurement was performed by two physicians (ML, EP).

\section{Obtaining en face OCT C-scan images}

En face OCT images were obtained from the macular cube, by selecting the 'advanced visualization' mode in the analysis list of Cirrus software. This interactive multiplanar reformatting enables a three-dimensional view of image cross-sections. Cube scan analyzes incorporate an algorithm to automatically find and display the ILM and the RPE. Three segmentations are possible: ILM, horizontal and RPE. Selecting one of these segmentations of the $X$-scan changes the en face image of the Z-scan. To improve image quality, grayscale of slices was preferred.

The three possible segmentations were tested to obtain the easiest-to-use and best en face image quality:

1. ILM slab segmentation: the inner reference line follows the ILM contour and is automatically detected by the device. In case of $\mathrm{MH}$, the discontinuity of this line creates an artifact line that does not always follow the $\mathrm{MH}$ walls. To obtain a full-thickness slab, the outer reference line of which the shape was parallel to the inner line, was placed manually at the $\mathrm{MH}$ base (Figure 2a).

2. Horizontal slab segmentation: the inner and outer reference lines are automatically defined as horizontal on the mapping area (Figure $2 b$ ).

3. RPE slab segmentation: in this segmentation, the outer reference line is automatically defined as the RPE line. The inner reference line was moved at the top of $\mathrm{MH}$ edges that are automatically pictured by the ILM line. This allowed obtaining a vision that was strictly perpendicular to the retina and the en face images represented well $\mathrm{MH}$ aperture size (Figure 2c).

\section{Image processing}

En face OCT images were captured in JPEG format and analyzed using ImageJ software (Version $1.43 \mathrm{u}$, Wayne Rasband; National Institutes of Health, Bethesda, MA, USA). $\mathrm{MH}$ surface areas were measured by pixel counting. Each scan was converted into an 8-bit grayscale image. Cubes of $6 \mathrm{~mm} \times 6 \mathrm{~mm}$ were trimmed so that the scale was accurate (number of pixels on one side equal to $6 \mathrm{~mm}$ ). The threshold was adjusted to minimum to only show the darkest pixels (Image $>$ Adjust $>$ Threshold $>$ Minimum). A hyporeflective area, corresponding to the $\mathrm{MH}$ seen in front view, was identified in the built-in image as a black area. The surface area was then automatically calculated using the basic packages for particle analysis of the software. The areas obtained are expressed in $\mathrm{mm}^{2}$ (Figure 3).

The sequence of the measurement method is summarized in Figure 4.

\section{Diameter and surface area comparisons}

The diameters measured on B-scans by the two observers were first compared with detect any bias. Horizontal $(H)$ and vertical $(V)$ diameters measured on the B-scans were compared. Then, from the measured surface areas, the mean $\mathrm{MH}$ diameter was calculated using the (diameter $=2 \times$ $\sqrt{ }(\operatorname{area} / \pi))$ formula. These mean diameters were then compared with those measured manually on the horizontal and vertical B-scans using the $((H+V) / 2)$ formula.

Surface areas measured on en face OCT were compared to the $\mathrm{MH}$ surface areas calculated using the horizontal and vertical diameters measured on the B-scans as follows: $\pi \times((H+V) / 4)^{2}$. 


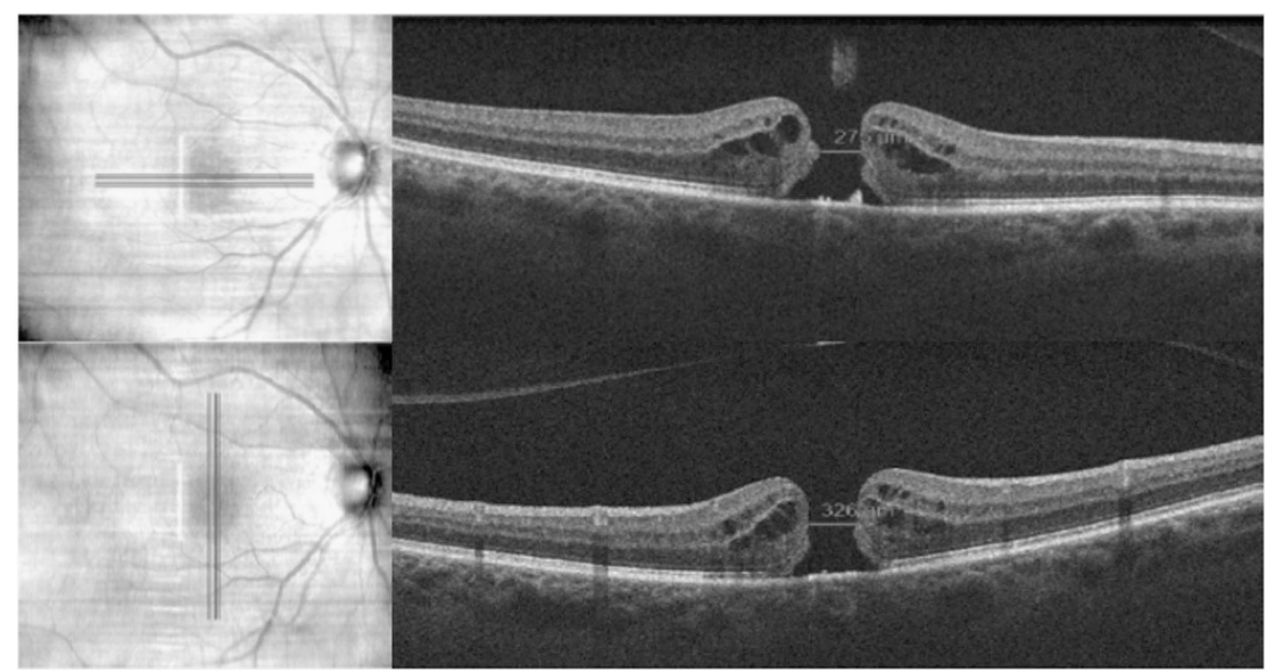

Figure 1 Macular hole diameter measured on horizontal and vertical SD-OCT B-scans. The diameter is measured with a caliper as a line drawn roughly parallel to the retinal pigment epithelium, at the narrowest distance between the hole edges.

\section{Statistical analysis}

Results are presented as a mean \pm standard deviation for continuous variables and as proportions (\%) for categorical variables. The distribution pattern of the included variables was compared to a theoretical normal distribution using a Kolmogorov-Smirnov test. Interobserver agreement for the measurement of $\mathrm{MH}$ vertical and horizontal diameters using B-scan highdefinition raster lines was assessed by the intraclass correlation coefficient (ICC), and the limits of agreement were assessed using the Bland-Altman method. The ICC was considered reliable if the values ranged between 0.4 and 0.75 , and values $>0.75$ were considered excellent. The paired Student $t$-test was used to compare continuous data. Linear regression, with determination of the Pearson coefficient, was used to analyze associations between two continuous variables. The Bland-Altman method was also used to calculate the bias. Corrected $P$-values $<0.05$ were considered statistically significant. Statistical analysis was carried out using SPSS for Windows version 20.0 (SPSS, Inc, Chicago, IL, USA).

\section{Results}

Of the 37 patients imaged with the Cirrus, 19 had a macular cube acquisition with a signal strength $>5 / 10$ and were analyzed. Patient mean age at baseline was $72 \pm 8$ years (56-86). The best-corrected visual acuity was $0.72 \pm 0.34$ LogMAR (20/100 Snellen). Four eyes (21\%) had MH with vitreomacular traction and 15 eyes (79\%) had no vitreomacular traction. Patient characteristics are presented in Table 1.

\section{Segmentation method}

Among the three tested en face segmentations, the ILM slab segmentation could not provide a well-defined $\mathrm{MH}$ area on en face images in 9 out of the 19 cases (47\%). In these cases, the eccentric path of the outer reference line resulted in a hyperreflective annular area corresponding to the RPE reducing the MH to a hyporeflective spot in the center of the hole on the en face image. This artifact precluded the further automated measurement of the $\mathrm{MH}$ area and the manual adjustment of the ILM line was not possible (Figure 2a). The horizontal slab segmentation could not provide an appropriate en face image when the retinal plane was not strictly parallel to these lines, as observed in 6 out of the 19 cases (32\%) and shown in Figure $2 b$. Also images could not be aligned manually. The RPE slab segmentation allowed obtaining a welldefined en face image in 15 out of the 19 cases (79\%), as shown in Figure 2c. In the remaining five patients, an adjustment was required because the device hardly located the ILM owing to the presence of a vitreomacular traction. In four of these cases, the inner reference line was positioned manually on the $\mathrm{MH}$ edge top. In the remaining case, the inner reference line could not be raised to the ILM owing to the position of the image in the mapping area. In this case, the inner reference line was positioned on the innermost possible area, in order to cover the aperture size location. The manual adjustment of the position of the inner reference line allowed analyzing all cases (100\%).

Of the three assessed methods, the RPE slab segmentation was the easiest-to-use method with the best feasibility (79\% and 100\% without and with adjustment, respectively) compared with the ILM slab segmentation (53\%) and Horizontal slab segmentation (68\%). 

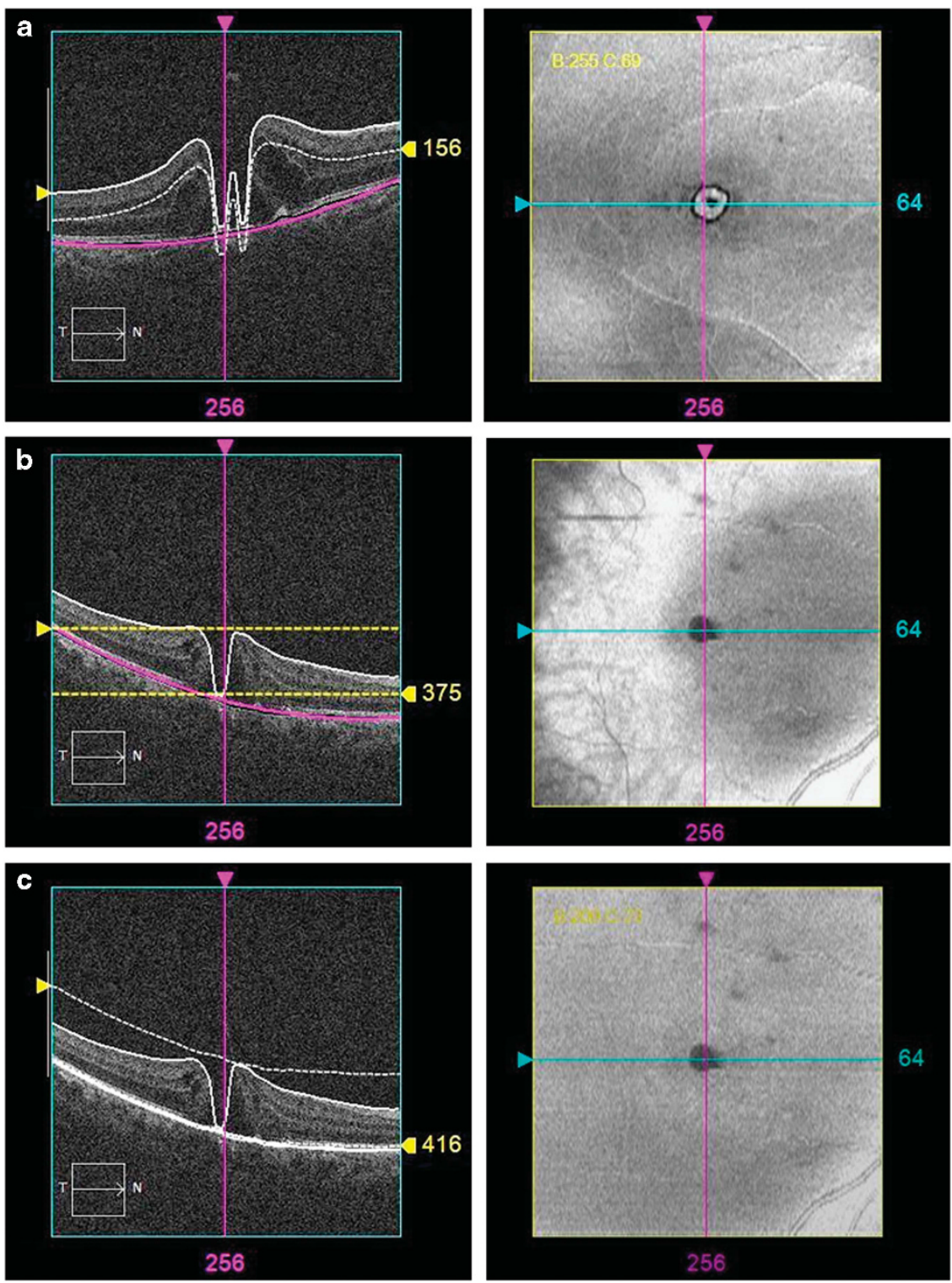

Figure 2 Possible segmentations in en face analysis on Cirrus 5000 HD-OCT. (a) Internal limiting membrane (ILM) slab segmentation. The line of the ILM does not follow the MH shape, and shows an eccentric pattern. As a result, the en face images obtained with the ILM segmentation show a hyperreflective annular area corresponding to the retinal pigment epithelium (RPE) and a hyporeflective spot corresponding to the remaining MH center. (b) Horizontal slab segmentation in the en face analysis does not align with the retinal shape, resulting in an unusable en face image. (c) RPE segmentation showing the inner line tangent to the ILM display, and the outer line delineating the RPE. En face images show a well-defined dark spot, suitable for surface area measurement.

Therefore, we chose to use the en face images obtained with the RPE slab segmentation to measure $\mathrm{MH}$ surface areas.

\section{Diameter and surface area comparison}

When comparing horizontal and vertical diameters measured on the B-scans, a perfect agreement was observed between the two vitreoretinal specialist observers (EP and ML). The ICC was excellent for both variables (0.983 for horizontal diameter and 0.968 for vertical diameter). There was no substantial bias between the two observers for both variables (bias $=-1.32$ for horizontal diameter and 7.21 for vertical diameter). A mean absolute difference of $54 \pm 47 \mu \mathrm{m}(0-180)$ was observed between the horizontal and vertical diameters but it did not reach significance (paired $t$-test, $P=0.874$ ). The horizontal diameter was larger than the vertical diameter in $10 / 19$ cases (53\%). However, as in $42 \%$ of cases $(8 / 19)$ this difference was $>50 \mu \mathrm{m}$, we decided to 


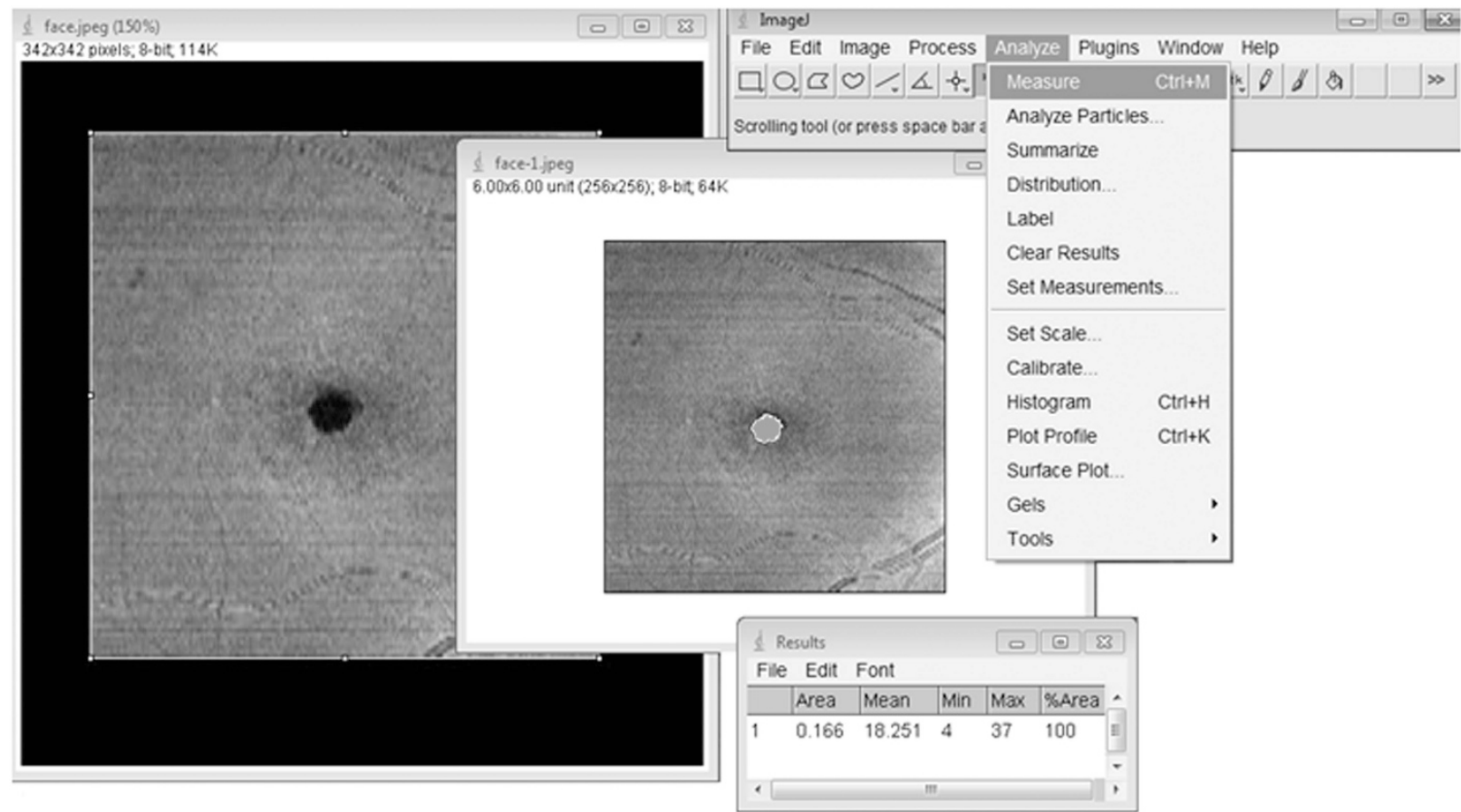

Figure 3 Image processing and surface area measurement with ImageJ with adjustment of the threshold to minimum.

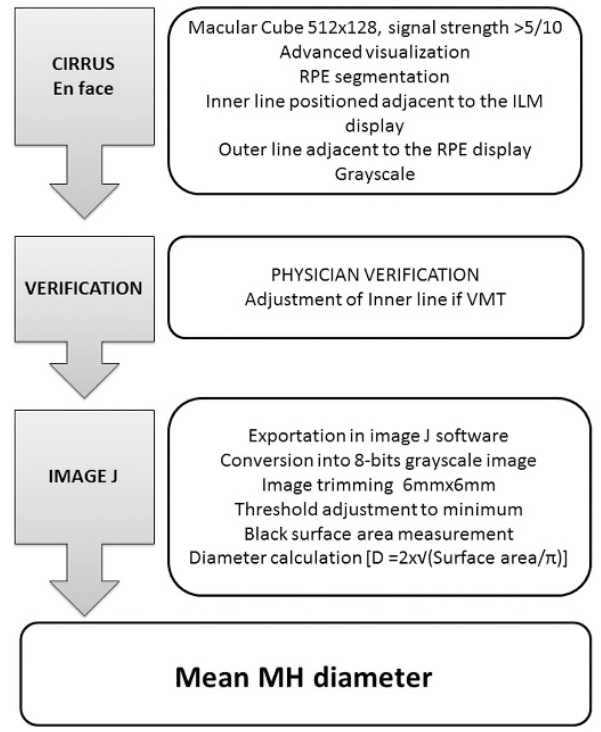

Figure 4 Measurement method using en face imaging from macular cube acquisitions to mean macular hole diameter.

calculate a mean measured diameter $((H+V) / 2)$ and found a value of $418 \pm 142 \mu \mathrm{m}$ (202-676) (Supplementary Figure S1).

The mean MH diameter calculated from the surface areas was $435 \pm 143 \mu \mathrm{m}(195-693)$ and did not significantly differ from the mean measured diameter (paired $t$-test, $P=0.168, r^{2}=0.88$, bias $=16.96$ ). In addition, no significant difference was found between the
Table 1 Baseline characteristics of patients with macular hole

\begin{tabular}{lc}
\hline $\mathrm{n}=19$ & \\
\hline Age (years) & $72 \pm 8(56-86)$ \\
Sex (M/F) & $3 / 16$ \\
Eye (R/L) & $13 / 6$ \\
Lens status (Phakic/ & $13 / 6$ \\
Pseudophakic) & \\
Initial VA (LogMAR) & $20 / 100$ \\
Duration of VA loss (days) & $204 \pm 245(15-1080)$ \\
OCT & \\
VMT /no VMT & $4 / 15$ \\
Diameter ( $\mu$ m) & $626 \pm 139(214-705)$ \\
ERM & $6(32 \%)$ \\
CMT ( $\mu$ m) & $347 \pm 52(239-426)$ \\
Surgery & \\
Combined with cataract & $7(37 \%)$ \\
ILM peeling & $14(74 \%)$ \\
Closure rate & $19(100 \%)$ \\
\hline
\end{tabular}

Abbreviations: CMT, central macular thickness; ERM, epiretinal membrane; ILM, Internal limiting membrane; LogMAR, logarithm of the minimum angle of resolution; OCT, optical coherence tomography; VA, visual acuity; VMT, vitreomacular traction. Quantitative values are presented as a mean $\pm \mathrm{SD}$ (range).

horizontal and mean $\mathrm{MH}$ diameters (paired $t$-test, $P=0.482)$ with a good correlation $\left(r^{2}=0.86\right.$, bias $\left.=8.83\right)$ and between the vertical and mean MH diameters (paired $t$-test, $P=0.133, r^{2}=0.79$, bias $=25.09$ ). Correlation curves and biases are detailed in supplementary material 
(Supplementary Figure S2). The minimal bias was observed for the comparison between the calculated and horizontal diameters $(8.83 \mu \mathrm{m})$. The mean difference between the mean $\mathrm{MH}$ diameter calculated from the surface areas and the mean measured diameter was $39 \pm 36 \mu \mathrm{m}(6-140)$.

Comparison and correlation of measured and calculated diameters and surface areas are detailed in Supplementary Table S1 and no significant difference was observed.

\section{Discussion}

In a series of 19 eyes with primary $\mathrm{MH}$, we showed that en face SD-OCT analysis allowed a feasible and accurate semi-automated measurement of $\mathrm{MH}$ size. Nowadays, OCT analysis is the gold standard for the diagnosis, characterization, monitoring and therapeutic decision of MH. ${ }^{2,13}$ Preoperative OCT is commonly used to assess the vitreous status and measure $\mathrm{MH}$ size, all these parameters being needed for the IVTSG classification of $\mathrm{MH}^{2}$ The horizontal OCT cross-section B-scan passing through the fovea center or across the largest $\mathrm{MH}$ width is typically used for clinical evaluation. The horizontal line is commonly used by convention but also by convenience because most devices provide horizontal scans and also because it allows imaging the vitreous attachment to the optic disc. The diameter is measured manually with a caliper as a line roughly parallel to the RPE at the narrowest point in the mid retina. This measurement method presents several biases and difficulties: first, it reduces $\mathrm{MH}$ to a perfect regular circle and cylinder centered on the fovea. This may lead to a misjudgment of $\mathrm{MH}$ size given that $\mathrm{MH}$ largest axis is not always horizontal. In our series, the difference between the horizontal and vertical diameters, although not significant, was extremely variable from one patient to another ranging from $-189 \mu \mathrm{m}$ to $+122 \mu \mathrm{m}$, and was variably positive or negative. This finding could support the concept that the true largest width of $\mathrm{MH}$ could be horizontal, vertical or lie in an oblique axis or could be only related to measurements. Moreover, the mean absolute difference was of about $50 \mu \mathrm{m}$. This difference represented one tenth of $\mathrm{MH}$ size, which could explain why it did not reach significance and that $\mathrm{MH}$ are often considered as round. However, taken individually such a difference could be clinically significant as the therapeutic decision is influenced by the cutoffs of 250 and $400 \mu \mathrm{m}$. Second, to be accurate, this measurement method requires that the horizontal scan passes exactly through the center of the $\mathrm{MH}$, which cannot be sure and depends on the line spacing and position. Finally, this method is manual; therefore, its reproducibility and precision depend on the observer's experience. Indeed, the diameter should be the narrowest distance between the edges, which requires an experimented eye in some cases. In our series, manual measurements were performed by two experienced vitreoretinal specialists. This human factor is known and in order to illustrate its impact, $\mathrm{MH}$ diameters of the study population were also blindly measured by our imaging technician. The mean absolute difference between the $\mathrm{MH}$ diameters measured by her and the vitreoretinal specialists was $57 \pm 62 \mu \mathrm{m}$ with extreme differences going up to $259 \mu \mathrm{m}$.

In contrast, surface area measurements performed on en face images do not depend on the variability of $\mathrm{MH}$ shape or the examiner's experience. The mean $\mathrm{MH}$ diameter extracted from the surface areas is less biased than horizontal or vertical diameters measured on a single B-scan, especially in cases of oval or irregular $\mathrm{MH}$. Indeed, en face images of $\mathrm{MH}$ are more similar to the anatomic reality and to what is observed on fundus examination.

In our series, the segmentation that provided the best defined en face images was the RPE slab segmentation as the inner and outer reference lines were automatically defined, and reliable images were obtained in $79 \%$ of cases, and in $100 \%$ after adjustment of the position of the inner reference line. The cases for which adjustment was required were those presenting with vitreomacular traction. In these cases, the MH shape could be asymmetrical, making its measurement on horizontal B-scans even more difficult. Thus, we considered that measuring $\mathrm{MH}$ size on en face OCT images could be an alternative option to the measurement of the horizontal diameter on OCT B-scans, in at least good image quality cases, especially if such a measurement method is integrated in the OCT software. In the era of multimodal imaging, measuring $\mathrm{MH}$ size using en face OCT takes up the current challenge to propose an automated measurement technique that is informative, reproducible, accessible and affordable in terms of material and time, so that it could be used in everyday clinical practice. Indeed with their hyporeflective cavity, MHs are well suited for automated segmentation and the high-contrast boundaries of the RPE and ILM make unequivocal and accurate the computerized delineation of boundaries. Furthermore, MHs are relatively constrained in their geometric shape and are always found in the foveal area, making easier the task of fully automated measurement devices. The analysis of the horizontal B-scan would be more complex to be automated. In this study, we suggested a semi-automated measurement of $\mathrm{MH}$ surface area using en face OCT C-scan images for which the complete automation would be easier to implement to obtain MH size (Figure 4).

One limitation of our study is its small size. We chose to only select good quality macular cubes (signal strength 
greater than 5/10) to avoid any quality bias. This first study aimed to assess the feasibility of this en face measurement method. In some cases, the full automation could not be carried out because the presence of vitreomacular traction altered the automatic display of the ILM. This issue could be resolved by repositioning manually the ILM display, the following steps remaining automated. However, on the B-scans in these cases, the aperture size is not easy-to locate owing to the shape of the inner part of the hole, whereas en face imaging, once the inner line is repositioned, offers an easy measurement of MH size.

In conclusion, measuring $\mathrm{MH}$ surface area on en face OCT C-scans provides a mean $\mathrm{MH}$ diameter comparable to that measured on B-scan sections, which is currently the reference method. This technique allows a reliable estimation of $\mathrm{MH}$ size, and eliminates the potential biases related to the manual measurement on the B-scans. Automation of this measurement method would be possible in clinical and everyday practice if its integration in the OCT device allows performing it routinely.

\section{Summary}

What was known before

- MH size is commonly measured on a single horizontal B-scan optical coherence tomography, reducing $\mathrm{MHs}$ to perfect rounds.

What this study adds

- Measuring MH size using en face optical coherence tomography is feasible and accurate.

- MH size using en face OCT is closer to the anatomic reality of MHs.

- Its automation on OCT devices is possible and would prevent the bias of the commonly used manual measurement method.

\section{Conflict of interest}

The authors declare no conflict of interest.

\section{Acknowledgements}

Data analysis and manuscript drafting (ML, EP, AC), statistical analysis (EP, MES), figures (EP, AE), manuscript review (AE, RT).

\section{References}

1 Gass JD. Reappraisal of biomicroscopic classification of stages of development of a macular hole. Am J Ophthalmol 1995; 119(6): 752-759.

2 Duker JS, Kaiser PK, Binder S, de Smet MD, Gaudric A, Reichel E et al. The International Vitreomacular Traction Study Group classification of vitreomacular adhesion, traction, and macular hole. Ophthalmology 2013; 120(12): 2611-2619.

3 Tadayoni R, Svorenova I, Erginay A, Gaudric A, Massin P. Decreased retinal sensitivity after internal limiting membrane peeling for macular hole surgery. $\mathrm{Br} J$ Ophthalmol 2012; 96(12): 1513-1516.

4 Haller JA, Stalmans P, Benz MS, Gandorfer A, Pakola SJ, Girach A et al. Efficacy of intravitreal ocriplasmin for treatment of vitreomacular adhesion: subgroup analyses from two randomized trials. Ophthalmology 2015; 122(1): 117-122.

5 Ip MS, Baker BJ, Duker JS, Reichel E, Baumal CR, Gangnon R et al. Anatomical outcomes of surgery for idiopathic macular hole as determined by optical coherence tomography. Arch Ophthalmol 2002; 120(1): 29-35.

6 Ullrich S, Haritoglou C, Gass C, Schaumberger M, Ulbig MW, Kampik A. Macular hole size as a prognostic factor in macular hole surgery. Br J Ophthalmol 2002; 86(4): 390-393.

7 Kang SW, Ahn K, Ham D-I. Types of macular hole closure and their clinical implications. Br J Ophthalmol 2003; 87(8): 1015-1019.

8 Rosen RB, Hathaway M, Rogers J, Pedro J, Garcia P, Dobre GM et al. Simultaneous OCT/SLO/ICG imaging. Invest Ophthalmol Vis Sci 2009; 50(2): 851-860.

9 Greven MA, Elkin Z, Nelson RW, Leng T. En face imaging of epiretinal membranes and the retinal nerve fiber layer using swept-source optical coherence tomography. Ophthalmic Surg Lasers Imaging Retina 2016; 47(8): 730-734.

10 Matet A, Savastano MC, Rispoli M, Bergin C, Moulin A, Crisanti $\mathrm{P}$ et al. En face optical coherence tomography of foveal microstructure in full-thickness macular hole: a model to study perifoveal Müller cells. Am J Ophthalmol 2015; 159(6): 1142-51.e3.

11 Alkabes M, Salinas C, Vitale L, Burés-Jelstrup A, Nucci P, Mateo C. En face optical coherence tomography of inner retinal defects after internal limiting membrane peeling for idiopathic macular hole. Invest Ophthalmol Vis Sci 2011; 52(11): 8349-8355.

12 Philippakis E, Amouyal F, Couturier A, Boulanger-Scemama E, Gaudric A, Tadayoni R. Size and vitreomacular attachment of primary full-thickness macular holes. Br J Ophthalmol 2017; 101(7): 951-954.

13 Gaudric A, Haouchine B, Massin P, Paques M, Blain P, Erginay A. Macular hole formation: new data provided by optical coherence tomography. Arch Ophthalmol 1999; 117(6): 744-751.

Supplementary Information accompanies this paper on Eye website (http://www.nature.com/eye) 